\title{
The token's secret: the two-faced financial incentive of the token economy
}

\author{
Benedict J. Drasch ${ }^{1,4}$ (D) Gilbert Fridgen $^{2} \cdot$ Tobias Manner-Romberg $^{3} \cdot$ Fenja M. Nolting ${ }^{3} \cdot$ Sven Radszuwill ${ }^{1,4}$
}

Received: 16 July 2019 / Accepted: 19 February 2020 / Published online: 13 March 2020

(C) The Author(s) 2020

\begin{abstract}
Multi-sided platforms are omnipresent in today's digital world. However, establishing a platform includes challenges: The platform utility usually increases with the number of participants. At an early stage, potential participants expect the platform utility to be low and lack an incentive to join (i.e., "chicken and egg" problem). Blockchain-enabled utility tokens hold the promise to overcome this problem. They supposedly provide a suitable financial incentive for their owners to join the platform as soon as possible. In the first half of 2018 , investors seemed to believe in the presumption and spent more than US\$17.6 billion in token sales. To date, we know little about this financial incentive in the context of the token economy. For this purpose, we model the token value development and the associated incentives in a multi-sided blockchain-enabled platform. The resulting findings suggest that blockchain-enabled utility tokens can help to overcome the "chicken and egg" problem. However, these tokens lead to contradictory incentives for platform participants, and can even inhibit platform usage. The contribution of our work is twofold: First, we develop one of the first models for token value development. Second, our research contributes to a deeper understanding of the utility token's financial incentive.
\end{abstract}

Keywords Multi-sided platform $\cdot$ Platform adoption $\cdot$ Blockchain $\cdot$ Tokenization $\cdot$ Token valuation

JEL classification $\mathrm{G} 15 \cdot \mathrm{G} 23 \cdot \mathrm{O} 33$

Responsible Editor: Val Anne Hooper

Benedict J. Drasch

benedict.drasch@fim-rc.de

Gilbert Fridgen

gilbert.fridgen@uni.lu

Tobias Manner-Romberg

tobias.manner-romberg@tum.de

Fenja M. Nolting

fenja.nolting@tum.de

Sven Radszuwill

sven.radszuwill@fit.fraunhofer.de; sven.radszuwill@ fim-rc.de

1 Project Group Business \& Information Systems Engineering of Fraunhofer FIT, Wittelsbacherring 10, 95444 Bayreuth, Germany

2 SnT - Interdisciplinary Centre for Security, Reliability and Trust, University of Luxembourg, 29 Avenue John F. Kennedy,

1855 Luxembourg, Luxembourg

3 FIM Research Center, University of Augsburg, Universitaetsstr. 12, 86159 Augsburg, Germany

4 FIM Research Center, University of Bayreuth, Wittelsbacherring 10, 95444 Bayreuth, Germany

\section{Introduction}

Multi-sided platforms (MSPs) such as Amazon, Uber, or Airbnb are among the most important players within the global economic activity. According to a survey by "The Center for Global Enterprise", in 2016, 160 MSPs existed, comprising a total market cap of US\$ 1.1 trillion (Evans and Gawer 2016). MSPs, also referred to as "matchmakers", act as an intermediary bringing together two or more sides of the market, typically sellers and buyers (Armstrong 2006; Rochet and Tirole 2003). Thus, MSPs create value by reducing search costs and shared transaction costs (Hagiu 2007). This value depends on the size of the platform, as the utility a participant derives from using the platform depends on the number of other participants on the platform. The well-known network effects describe this phenomenon (Katz and Shapiro 1985; Liebowitz and Margolis 1994).

Once the platform reaches a critical mass of users, these network effects take effect and accelerate platform growth (Evans 2014; Oren and Smith 1981), enabling a completely new path for growth to companies (Shapiro and Varian 1999). 
Therefore, developing platform business models became a focus topic for many companies in the past few years. However, the first platform users experience low platform utility, which is why potential users lack the incentive to join the platform at an early stage. Especially in two-sided markets, buyers have little incentive to join a platform if no sellers are present and vice versa, which is also called the "chicken and egg" problem (Caillaud and Julien 2003). A company that intends to establish a platform has to overcome this problem, which is an expensive and slow endeavour since a suitable incentive for early platform adopters is necessary (Evans 2009).

In recent years, a new technology emerged that may be able to change the incentives for platform participation and to overcome the initial "chicken and egg" problem: the blockchain technology. Satoshi Nakamoto introduced the blockchain in 2008 in a publication on a peer-to-peer electronic cash system, the bitcoin blockchain (Nakamoto 2008). It has since been adopted and further developed to fit a variety of use cases, such as blockchain-based platforms. The entity that builds up a blockchain-based platform can consist of one or many individuals, companies, or organizations. Some examples of these platforms include Ethlance, a marketplace for jobs (Urgo et al. 2017), Golem, a marketplace for computing power (Zawistowski et al. 2016), or DataBroker, a marketplace for sensor data (van Niekerk and van der Veer 2018). The entity behind the platforms issues its own blockchain-enabled token (Bachmann et al. 2019; Fridgen et al. 2018; Lee et al. 2018). So far, various token designs enable different applications. One token type is the "utility token", which represents the right to access the offered products or services on the platform (ICOscoring 2018). In the blockchain context, we refer to this representation as tokenization (Dale 2018).

Currently, the blockchain community ascribes many positive characteristics to utility tokens. In the following, we describe the presumptions the blockchain community makes in blogs and whitepapers regarding a postulated financial incentive of utility tokens and its impact on platform development. Utility tokens are seen as the de facto means of payment on the platform (Wesley 2018; Wilmoth 2018). Before the launch of the platform, the entity issues the tokens at a certain price and uses the proceeds of the sale as an alternative funding to finance the platform development (Chuen et al. 2017; Ehrsam 2016a). The issuing entity promotes the token sale with the prospect of an increase of the token value. It states that rising numbers of platform participants lead to an increase in demand for the tokens. As the entity limits the supply of tokens, an increase in demand is then said to lead to a higher token value (Karnjanaprakorn 2017; Wesley 2018). Hence, buying tokens earlier provides a financial incentive since the token value presumably increases. Thus, the characteristic of the tokens is not only restricted to a means of payment, but they are also seen as an investment vehicle (Aru 2018), supposed to give early adopters a compensation (Karnjanaprakorn 2017).

Based on this presumptions, the blockchain community draws the following conclusions: The potential financial gain from investing early into tokens can reflect a suitable financial incentive to overcome the above-mentioned "chicken and egg" problem (Dixon 2017; Ehrsam 2016a). Potential users follow the incentive to become an early part of the platform and benefit from platform growth, e.g., profit from the increasing token value. This permanent token value upside potential is associated with positive effects on platform growth: the socalled token network effects (Dixon 2017; Karnjanaprakorn 2017). At this point, every participant of the platform has an incentive to increase platform growth, and thus profit from an increase of the token value (Karnjanaprakorn 2017). Erickson (2018) and Karnjanaprakorn (2017) assume this effect to lead to faster platform growth and even to bigger platforms.

In the well-established research stream of MSPs, blockchain-enabled tokens depict a novel phenomenon that seems to introduce new dynamics in the process of platform development. In this intersection, research only started to analyse the interplay of existing knowledge and open research questions (e.g., Chod et al. 2019; Kristoufek 2013; Reid and Harrigan 2013). The majority of articles analysing token economics and blockchain-based platforms are blogs and white papers. Nevertheless, multi-billion-dollar industries base their decisions on existing knowledge in the realm of token economics and mechanisms of blockchain-based platforms. Especially the potential financial incentive demands for a critical assessment of the supposed phenomenon. To date, studies in this context are sparse. In this context, the paper at hand is a first attempt to challenge the assumptions that utility tokens enable platform-growth. The impact of tokenization and the token value upside potential on blockchain-based platforms is not only relevant for the scientific community, but also for practitioners and potential investors. Against this backdrop, we pose the following research question:

Can utility tokens issued on a blockchain-based platform hold a financial incentive? And if so, what are the implications for the adoption of these platforms?

To answer this research question, we model user incentives in a two-sided blockchain-based platform where users (sellers and buyers) interact to trade one certain good. The value development of the platform's token is the underlying factor determining user incentives. We divide our model into two distinct phases to investigate the value development over time. In the first phase, $\mathrm{P}_{1}$, users of the platform can only buy tokens, but are not able to access the platform, e.g., the good. We apply a qualitative-argumentative approach to explain changes in token value and assess their impact on platform adoption. In the second phase, $\mathrm{P}_{2}$, the platform launches, and sellers and buyers start to trade. With the launch of the platform, the token becomes a means of payment. For $\mathrm{P}_{2}$, we apply a monetary 
approach to model the token value. We use this model to propose the conditions under which a financial incentive for token owners exists. As a result, we derive two major propositions for the financial incentive on the platform usage. Further, this paper is among the first to indicate that the supposed financial incentive is not what it seems to be, but has a two-faced character.

The remainder of the paper unfolds as follows. Section 2 sets the theoretical foundations of the platform economy and the token \& blockchain economy. In section 3, we describe our setting, model the token value and interpret the relation between changes of the token value and platform adoption. In section 4 , we provide an overall summary and discuss the implications of our results for theory and practice. The conclusion and proposed future research topics complete the paper.

\section{Theoretical foundation}

\section{Platform economy}

In the past years, several experts claimed an economic paradigm shift from linear business models towards digital platform economies (e.g., Brand 2017; Brousseau and Penard 2007; Kenney and Zysman 2016). These digital platforms, which only developed in the past decade, were promoted by the trend of digitalization and originate from non-digital platforms (de Reuver et al. 2018). Industrial innovation management research interpreted platforms as a stable core and a variable periphery (Baldwin and Woodard 2009). From this concept resulted various opportunities for distributed development and innovation, e.g., through modularisation (Baldwin and Clark 2000; Henderson and Clark 1990). This research stream has categorised platforms with their regard to the production process scope, and focused on platforms in terms of a vehicle for modularization, such as supply-chain platforms or capability exchange platforms (Gawer 2014). In contrast, the characteristics of digital platforms led to multiple settings of platform governance, so there is no single owner of the core anymore (Henfridsson et al. 2014; de Reuver et al. 2018). In the remainder of this paper, we focus on MSPs as enabler of direct interactions between two or more distinct sides, whereas each side is affiliated with a digital platform (Hagiu and Wright 2015). MSPs differ from other platform-like interactions between participants, such as a vertical integration (e.g., an organization operates and sells goods via a platform), reselling (e.g., an organization buys goods or services and sells them to a customer), or input supply (e.g., an organization buys goods from a supplier and sales them) (Hagiu and Wright 2015). MSP providers like Amazon mediate product or service interactions between different platform participants who need each other in some way (e.g., consumers and suppliers). Once established, MSPs impose high entry barriers on potential competitors (Kenney and Zysman 2016; Zhu and Iansiti 2012).

In recent decades, a large number of different MSPs developed in a variety of markets (Evans 2003; Hagiu 2007). If there are several platforms competing for the same market, consumers can participate in multiple platforms in order to obtain the maximal network benefits (Choi 2010). Research differentiates between the affiliation of participants to one (single-homing) or more than one platform (multiple-homing) (Rochet and Tirole 2003). A popular example for multi-homing is the market for credit cards that splits into various platforms (e.g., MasterCard, VISA): most of the merchants around the world accept more than one credit card, and most of the consumers carry at least one of the two. Consequently, both sides of the platform pursue multi-homing. In twoor multi-sided markets, multi-homing ties platforms together and increases the total surplus, as competition in the respective platforms affects each other and consumers access the content associated with it (Belleflamme and Peitz 2019; Choi 2010).

The challenge for all MSPs is to incentivize the various sides to join the platform. In MSPs, participants benefit from platform participation, and from direct and indirect network effects. Through their participation, participants can either sell or buy the underlying product or service (they are part of the platform). The direct effect is the value for a participant that results from other participants of the same side to participate on the platform as well (Shapiro and Varian 1999). Indirect network effects occur when the value obtained by one kind of platform participant (buyer) increases with the numbers of the other kind of platform participant (seller), or vice versa (Shapiro and Varian 1999). Consequently, platform owners face the challenge of low platform participation at an early stage of platform development, and accompanying low platform utility for (potential) platform participants.

To date, research in the realm of platform economy mainly focused on the perspectives of platform owners and sellers. For instance, Parker and van Alstyne (2009) describe six challenges in platform licensing and open innovation. Parker and van Alstyne (2018) analyse the challenge of the right degree of openness from a platform owners perspective in regard to platform developers' intellectual property rights. Further, research evaluates the effects of innovation on and within platforms (Evans et al. 2008; Hargadon 2008; Weitzman 1998). However, Blockchain technology is considered to change the established market rules of platform development (e.g., to overcome the "chicken and egg" problem), and therefore may disrupt the traditional platform economy (Karnjanaprakorn 2017; Lindman et al. 2017; Walter 2017). 
The opportunity to incorporate user incentives in the development phase and to apply token offerings can change traditional findings and calls for further analysis. So far, existing research has not addressed this presumption.

\section{Blockchain \& token economy}

Blockchain is a peer-to-peer protocol for transactional data, which is decentralized, transparent, time-stamped and shared across a network of untrusted participants (Xu et al. 2017). It is said to allow for disintermediation as intermediaries no longer need to verify transactions. In this network, trust in the underlying code and consensus rules replaces trustworthiness in the intermediaries (Catalini and Gans 2016). Those consensus rules are part of the underlying blockchain protocol and confirm each transaction on the particular blockchain (Zheng et al. 2017). There are several consensus mechanisms, e.g., "proof-of-work" and "proof-of-stake" (King and Nadal 2012; Xu et al. 2017).

In recent years, further blockchain protocols developed, such as the Ethereum blockchain (Buterin 2014). This socalled second generation blockchain provides a programmable infrastructure based on a Turing-complete programming language enabling "smart contracts" (Buterin 2014; Szabo 1997). Smart contracts are programs that allow different participants to carry out transactions securely as the correct execution of these programs is verified by a consensus protocol. One application of smart contracts is the possibility to issue tokens on top of a blockchain application (Beck et al. 2016; Catalini and Gans 2016; Xu et al. 2017).

Tokens are a medium of exchange used to purchase various goods, services, or privileges (Swan 2015). In a blockchain network, network participants use digital tokens for various purposes, e.g., as an internal unit of account, for the verification of block-writing, as a facilitation of transactions, or for more creative use cases such as preventing unintended use of the blockchain, or granting token owners access (Conley 2017; Fridgen et al. 2018; Glaser 2017; Schweizer et al. 2017)).

It is fundamental to distinguish between native tokens (e.g., Bitcoin) inherent to a blockchain protocol and on-chain tokens (e.g., Golem or DataBroker) issued on top of a blockchain using smart contracts (Buterin 2014; Kuo Chuen 2017). There are various types of on-chain tokens. In the following, we analyse asset-backed tokens and utility tokens, since these are the most common types (Asanov 2018; Pietrewicz 2017). Asset-backed tokens build cryptographic representation of traditional assets such as equity, gold, or even a fiat currency. Therefore, the token value is linked to the value of the underlying (Oliveira et al. 2018). Utility tokens on the other hand grant access to digital services or products (Michael et al. 2018). The tokens represent a means of payment on the platform and can be traded on secondary markets (Pietrewicz 2017). In contrast to cryptocurrencies like Bitcoin that serve as a cross-platform means of payment, the issuer of utility tokens limits their use to the respective platform (Katalyse.io. 2018).

The initial distribution of on-chain tokens can take place in several ways. First, the issuing entity can spread the tokens for free to owners of specific cryptocurrencies as part of its marketing strategy in a process called airdrop (Harrigan et al. 2018). Second, developers of a blockchain project can earn tokens as a giveaway (Ehrsam 2016b). Third, the issuing entity can distribute tokens in Initial Coin Offerings (ICOs) in exchange for a payment of fiat currency or cryptocurrency. Usually, the sale process serves to finance an underlying blockchain project (Conley 2017). ICOs became a popular alternative to traditional financing methods for organizations (Bachmann et al. 2019; Boreiko and Sahdev 2018; Fridgen et al. 2018; Li and Mann 2018; Schweizer et al. 2017).

In recent months, researchers started to evaluate the opportunity of said tokens to foster platform development. The idea is that early platform adopters can participate on the financial success of a platform. Bakos and Halaburda (2019) develop a model to investigate the use of tradable tokens to solve this coordination problem, and find that platform-specific tokens can aide to overcome the coordination problem in a platform development setting. Their findings indicate that the extraordinary reduction in transaction costs and the increase in functionality, awareness, popularity, and acceptance results in increasing application of these tokens. Li and Mann (2018) underpin this finding and warn against a universal ban of ICOs (e.g., China, South Korea), since tokens can serve as a part of the platform's operational process of building up user interactions. Chen (2018) analyses the potential democratization of entrepreneurship and innovation associated with blockchain tokens. He points out that the blockchain technology may enable entrepreneurs to raise funds directly, democratize access to financial capital, give investors the opportunity to invest in early-stage projects, restructure fundraising and investing, and facilitates user and developer communities.

\section{Modelling tokenization of the platform economy}

For the analysis of the potential financial incentive, we develop a simplified model of a blockchain-based platform, investigate the effects of tokenization in two distinct, consecutive phases $\left(\mathrm{P}_{1}, \mathrm{P}_{2}\right)$ of the developing platform, and interpret consequences on platform adoption.

\section{Model set-up}

Our subject of interest is the development of a platform that allows a two-sided market where buyers and sellers of a certain good (or service) interact. Behind the platform, a platform-building entity (e.g., one or multiple corporate or non-profit organizations) drives the development. The 


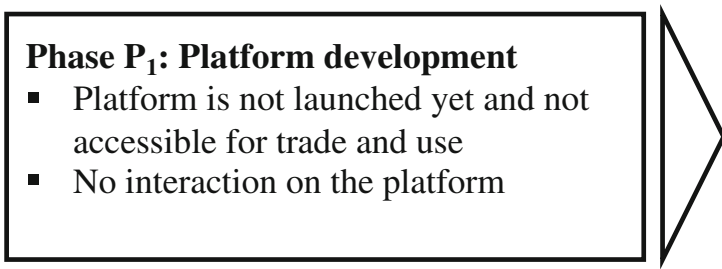

Fig. 1. Phases of platform development

platform is blockchain-based, which means that all platform transactions are stored on the underlying blockchain. Further, the platform has its own means of payment, a blockchainbased utility token (on-chain). For simplification, we restrict the number of goods on the platform to one. This good is also purchasable on markets outside of this platform (i.e., multihoming).

Before the platform is accessible to users, the platform building entity issues tokens with the aid of smart contracts (e.g., during an ICO. For a detailed description of design parameters for ICOs, please cf. Bachmann et al. 2019, Fridgen et al. 2018). The tokens are for sale in exchange for a fiat currency. The proceeds of the sale serve to finance the further development of the platform. The entity issues a fixed number of tokens that will not change at any later point in time (fixed token supply). The issued token is the only means of payment on the platform. Nevertheless, the token is convertible in exchange for a fiat currency or cryptocurrency on a secondary market. There are no transaction costs on the platform and on the secondary market. Furthermore, there is no interest rate.

In the following, we separate the analysis of token value development into two distinct phases to answer our research question. This is important to account for the change in the token characteristics between the two phases (cf. Fig. 1). In $P_{1}$, the platform is not launched and not accessible for users yet. Thus, the token is not a means of payment since no trade takes place on the platform. $\mathrm{P}_{2}$ begins with the launch of the platform. After this point in time, buyers and sellers can access the platform and exchange the good for the token. Hence, in $\mathrm{P}_{2}$ the token is a means of payment and thus valuated as such. Fig. 1 visualizes the phases $P_{1}$ and $P_{2}$

\section{$P_{1}$ - before launch}

$\mathrm{P}_{1}$ starts with the token sale. The issuing entity sets the initial token price at an appropriate level. ${ }^{1}$ At this point, the platform is still in the development phase, thus no trade of the good takes place on the platform yet. This raises the question why a potential platform user should purchase the token during this phase. This is important since the token has no inherent value just because of its technical creation (Glaser 2017). According

\footnotetext{
${ }^{1}$ Our analysis focuses on the token value development to derive implications on user incentives. Consequently, we consider the token's value changes. For this purpose, the initial token value is of inferior importance.
}

to Cong et al. (2018), the purchase decision of a token depends on both the expected platform utility and the expected future token price. If potential platform participants believe that the platform and the traded good are useful in the future and the price for a token is adequate, they buy tokens in the future. If they further believe that the token value increases, they buy the token at the earliest possible moment to profit from the increase of the token value.

The upside potential of the token value inevitably leads to the following question: How to determine the value development of a token, which cannot yet fulfil its intended purpose? To the best of our knowledge, there is no scientifically accepted approach for such a valuation. Since the token is not a means of payment before $\mathrm{P}_{2}$, a valuation as such (e.g., based on a monetary model) is inappropriate. Following this argumentation, we expect supply and demand to drive the token value. Since our model assumes fixed token supply, the token value will increase if the demand is higher than the supply.

As a result, we suspect a self-fulfilling prophecy: If sufficient potential users have confidence in the platform and the token, they buy tokens to be part of the platform and expect a financial profit, because the token value increases with the increasing number of platform participants. This finding is also in line with Lee et al. (2018), who find that aggressive first-day token subscriptions by institutional investors drive token sales and lead to successful ICOs. Fig. 2 visualizes the decision alternatives for potential token buyers.

\section{$P_{2}$ - after launch}

With the successful platform launch, the second phase of platform adoption begins. At this point, the good is tradeable on the platform and the token is exchangeable in return for the good. Thus, the token characteristic changes to a means of payment and needs to be valued as such. Therefore, we apply a monetary approach to determine the exchange rate between the token and a fiat currency.

This monetary approach to determine the exchange rate bases on the purchasing power parity (PPP) as well as the quantity theory of money (QTM). QTM links the money economy to the real economy and postulates a direct effect of money supply on the price level in an economy (Tsoulfidis 2008). In combination with the theory of PPP, monetary approaches use the QTM to determine the long run equilibrium 


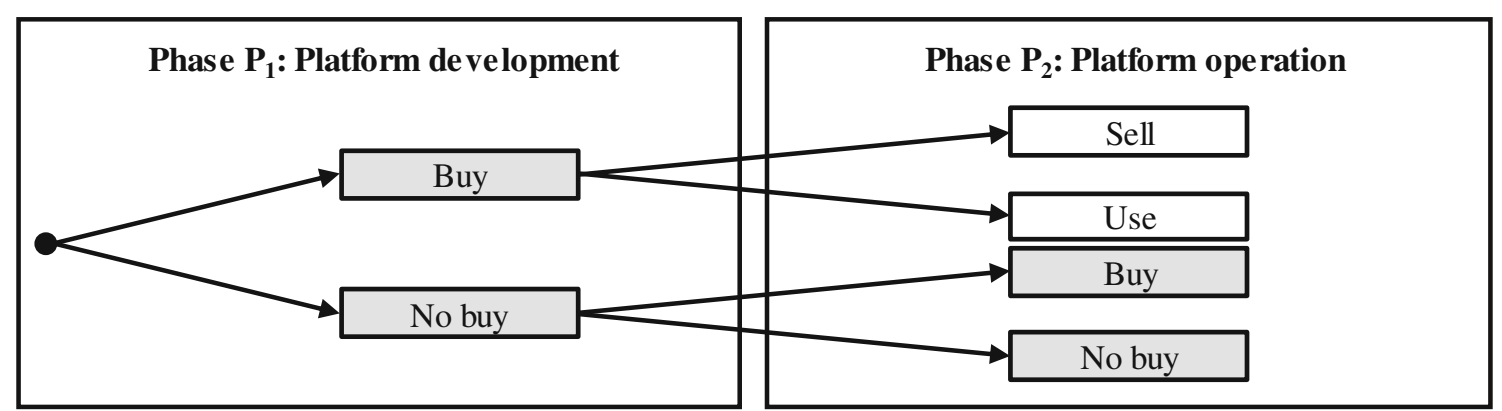

Fig. 2. Decision alternatives for potential token buyers

exchange rate, such as the flexible-price monetary model (Frenkel 1977). The application of this approach to the valuation of a utility token is in line with the recommendation of Vitalik Buterin, who proposes to apply the monetary equation of exchange to value a medium-of-exchange token (Buterin 2017). In many blog articles other authors follow this recommendation, using QTM to value a token (e.g., Beaman 2018; Burniske 2017; Evans 2018; Kilroe 2017).

In a first step, we consider the QTM equation of exchange, $M V=P Q$, where $M$ is the money supply, $V$ the velocity of the money circulation, $P$ is the price level, and $Q$ is the quantity of the resources provided (Newcomb 1913). The price level is expressed as:

$P=\frac{M V}{Q}$

Adapting this model to tokens, the definition of the variables changes as follows: $M$ is the total token supply, which is a fixed number; $V$ is the velocity of the token circulation, that is, the average number of transactions a token performs within a specified unit of time; $P$ is the price of the single good exchanged on the platform; and $Q$ is the amount of this good provided. It is important to note that $P$ is not the price for the token itself. The price of the token is expressed by an exchange rate $\varphi$ to a fiat currency. Following the theory of PPP, this exchange rate is:

$\varphi=\frac{P^{f}}{P}$

where $P^{f}$ is the price of the good outside of the blockchain-based platform measured in the fiat currency. Putting formula (1) and (2) together, the exchange rate $\varphi$ of a token to a fiat currency can be determined by:

$\varphi=\frac{P^{f} Q}{M V}$

As such, we express the token value by four parameters: three internal to the characteristics of the blockchain-based platform and one external to the platform, the platformexternal price of the good measured in a fiat currency.

Based on this definition of the token value $\varphi$, we consider the impact of a marginal token value change. To do so, we split $\mathrm{P}_{2}$ into two distinct periods $\left(t_{1}, t_{2}\right) \cdot \varphi$ can only change from the first to the second period, i.e., is constant in $t_{1}$ and $t_{2}$. Thus, a change in value from one period to the other can be ascertained by:

$\Delta \varphi=\varphi_{t_{2}}-\varphi_{t_{1}}$

If $\Delta \varphi$ is positive, the token owner profits from a financial gain, hence, has an incentive to hold the token (i.e., keep it as an investment). If $\Delta \varphi$ is negative, the token value decreases and the token owner has no incentive to hold the token.

In the following, we analyse under which circumstances $\Delta \varphi>0$, and thus, the token holds a financial incentive. For this purpose, we look at changes in one of the parameters of $\varphi$, while holding the other three values constant. $\Delta \varphi>0$ if $Q$ rises, ceteris paribus, i.e., when the amount of the good traded on the platform increases from $t_{1}$ to $t_{2}$. This is the case when more participants join the platform, and thus more trade takes place. $\Delta \varphi$ is also positive when $P$ rises, ceteris paribus, e.g., because the price level of a foreign market decreases, or $V$ decreases, ceteris paribus, e.g., due to external shocks (for a more detailed discussion of the QTM and its components, please refer to Friedman 2017; Woodford 2011). Since $M$ is fixed, it has no influence on $\Delta \varphi$.

To assess whether $\Delta \varphi$ affects platform adoption we determine the platform usage. The platform usage depends on the purchase decision of every buyer for the traded good on the platform. Therefore, we take a closer look at the purchase decision in $t_{1}$ of a buyer who already holds tokens. A purchase decision usually depends on the willingness to pay $w$ for the good and on the price $P$ of the good. $P$ is the price measured in tokens the buyer has to pay on the platform, not to be confused with $P^{f}$. If $w \geq P$, a utility maximizing consumer buys the good. However, on a tokenized platform, the purchase decision does not only depend on $w$. As the means of payment on the platform is the token, the buyer of the good has to use this token to pay for the good. Therefore, the buyer must also consider whether he is missing a potential financial gain if he uses the token to buy the good. Hence, the dependencies of the purchase decision on a tokenized platform change as follows:

$w-x \Delta \varphi \geq P$,

with $x$ representing the number of tokens needed to pay for the good. 
The following states occur:

- If $\Delta \varphi=0$, there is no incentive to hold the tokens and the purchase decision solely depends on $w$ and $P$.

- If $\Delta \varphi<0$, the token owner would suffer a loss of value of his tokens. Therefore, the token owner has a higher incentive to buy the good in $t_{1}$ than in $t_{2}$.

- If $\Delta \varphi>0$, the token owner benefits from an increase in token value, which is tantamount to a financial incentive to hold the tokens. Hence, the token owner faces the question whether to keep the tokens and benefit from this financial gain, or to use the tokens to buy the good while losing the potential gain. Thus, if $\Delta \varphi>0, w$ is reduced by $\Delta \varphi$. A reduction in the willingness to pay leads to a lower demand in the market overall and to a decline in platform usage.

If PPP holds, an increase in $\varphi$, and thus a positive $\Delta \varphi$ leads to a higher purchasing power in $t_{2}$. In other words, this corresponds with a decline in the price level for the good and thus to the typical characteristics of deflation. Following this analysis, the financial gain for the token owner associated with a positive change in token value has a negative effect on the platform overall. As the token owner decides between holding and using the token, the financial incentive to hold the token lowers the willingness to pay for the good. Thus, the overall demand for the good declines and results in a negative effect for sellers. This implies that a growth in trading volume from $t_{1}$ to $t_{2}$ (i.e., platform usage) leading to a higher token valuation has a direct negative impact on demand for the good in $t_{1}$.

\section{Demonstration}

In the following example, we aim to provide further illustration of the model application: Let's consider a platform that allows users to transfer money to other users of the platform. In exchange for every transaction, the user is charged one token. The platform building entity issues the tokens before the launch of the platform and sets an initial token price. Potential users become buyers of these tokens under the following conditions: First, buyers expect the transferral of money via this platform to be useful in the future. Second, the token price is adequate. Third, buyers expect the token price to rise in the near future, as they would otherwise defer their purchase until the platform launch. If these conditions result in a token purchase, a supply and demand consideration leads to the conclusion that the value of the token will increase. Therefore, even before the token is usable to transfer money via the platform, its value increases due to the fact that buyers believe in the growth of the platform.

After the launch of the platform, a token owner has three options: First, use the token to transfer money. Second, sell the token. Third, hold the token. A utility maximizing token owner has to weigh the benefits of a transaction via the platform against the upside potential of the token value. As monetary theory implies, the token value increases inter alia, if more users join the platform and more transactions take place. If the token owner believes in further platform growth, his potential financial gain in the future from selling the token at a higher price outweighs his benefits from using the token in the present at some point. If this consideration applies to multiple platform users, the expectation of future platform growth will hinder platform activity.

\section{Overarching results and implications}

In the following, we present two propositions that we derived from the observable phenomena and emphasize on the resulting implications for theory and practice.

Proposition 1: The blockchain-enabled tokenization of MSPs offers a financial incentive for platform users during platform development.

Our approach suggests a positive financial incentive in $\mathrm{P}_{1}$. If enough platform participants believe in a positive token value development, the self-fulfilling prophecy seems to overcome the "chicken and egg" problem. Expecting a financial profit, the assumed value increase incentivizes potential platform participants to buy tokens, and thus to join the platform in $\mathrm{P}_{1}$. Therefore, the platform obtains a first user base without the need for high investments by the platform building entity. The entity even receives financing for the platform development through the collected capital. Furthermore, the token owners have an incentive to grow the platform in order to profit from a rise in token value. Therefore, token owners and the entity work together toward a common goal: the appreciation of the platform and an increase of the token value. This leads to an alignment of incentives between the token owners and the entity. Nevertheless, a mere financial incentive to join the platform and accelerate platform growth can also have negative consequences. Initial studies suggest that the incentive structures described may encourage market manipulation such as "pump-and-dump" schemes (e.g., Corbet et al. 2018; Li and Mann 2018).

These findings for the platform development have relevant implications for research: First, the financial upside potential of tokens represents an incentive to join MSPs and thus extends existing literature on network effects and platform development in general. Second, the understanding of these additional incentive structures and dynamics need further sharpening in the future and thus offer a possible direction for future research. Regarding its practical implications, tokenization is of special interest to every platform building intention as it could potentially solve one of the major problems of platform development.

Proposition 2: The increasing number of platform participants results in an increase of the token value during platform operation. However, a utility maximizing consumer has to weigh the benefits of a transaction on the platform against the upside potential of the token value. 
If the token owner believes in further platform growth during platform operation, his potential financial gain from selling the token at a higher price in the future will at some point outweigh his benefits from using the platform. Based on this proposition, the expectation of future platform growth during platform operation will inhibit platform usage in the long run. This proposition is not in line with the proposition that existing research and blog articles follow. For the academic audience, these findings suggest that the incorporation of user incentives in the context of blockchain-based tokenization of MSPs is necessary. Our work expands the existing literature on MSPs by capturing financial utility alongside network effects. As our findings propose, the financial incentive is two-faced, and urgently needs further analysis for a deeper understanding. The research study at hand calls for evaluation of the token economy and can serve as a starting point. For the practice-oriented audience, our findings suggest that a careful application of token-financed platform development is necessary. Interestingly, the majority of the currently highest rated tokens are of deflationary design (Kaal 2018), which increases the relevance of our research contribution. We can only explain the dimensions of this phenomenon by assuming that most of the token issuing entities neglect the concepts of monetary theory, even though the intent of the token is a means of payment. Nevertheless, as Conley (2017) already pointed out, designing a successful token requires to consider common aspects of monetary theory, financial economics, and game theory. This includes a simple mechanism derived by the equation of exchange: the supply of money has to grow with the economic activity. Otherwise, the economy cannot grow, which reflects our results from $\mathrm{P}_{2}$. Hence, token issuing entities need to consider this proposition if they want to establish a sustainable economic cycle on their platform and avoid "pump and dump" schemes.

We emphasize that Proposition 2 neither reflects nor explains short-term market developments, e.g., it does not apply to exceptional market phases: First, a simultaneous increase in token value and platform usage can occur during a hype. However, first empirical analysis suggest that the median ICO destroys value of its token holders on average after half a year (Haffke and Fromberger 2018; Momtaz 2019). Second, Proposition 2 only applies if the financial incentive is the rationale for platform participation. It's conceivable to have a simultaneous increase in token value and platform participation for MSPs designed for illegal activities, which is a pressing issue in the blockchain community (Shoshitaishvili et al. 2014; Smith and Weismann 2014; van Hout and Bingham 2014). For instance, almost half of all bitcoin transactions have an illegal background (Foley et al. 2019).

Furthermore, we suspect a relation between Proposition 2 and Proposition 1 that we did not incorporate in our model. When users observe the same patterns across different platforms, this might also affect users trust in the platform development. Consequently, this observation can reduce the initially expected utility from platform participation, and further reduce the deflationary effect in $\mathrm{P}_{2}$. Future studies should analyse this effect with a focus on empirical data.

\section{Conclusion and future research}

Despite the growing interest from academia and practice in blockchain technology and tokens, theoretical knowledge on the effects of tokenization is scarce. Based on our research question, we investigated the implications of the application of blockchain-enabled tokens on the adoption of MSPs. For this purpose, we analysed the token value in two distinct phases of platform development and determined under which circumstances a token provides a financial incentive. To the best of our knowledge, the paper at hand is the first of its kind that distinguishes and analyses a possible financial incentive of tokens in different phases of platform development. This analysis is relevant for both theory and practice to approach further questions regarding the setup of ICOs.

We assess the financial incentive on platform adoption and provide a differentiated understanding of the effects of tokenization. Our findings imply that combining a financial incentive with a means of payment on a MSP leads to a contradictory effect: while the financial incentive first enables platform growth and helps to overcome the initial "chicken and egg" problem, it inhibits platform growth once the platform launches due to its deflationary character. We want to point out that the token value potentially decreases once the platform is accessible, as the token value's upside potential hinders the token usage. We believe that this study is theoretically and practically relevant, and hope that it provides fellow researchers with a foundation for continuing their research on blockchain technology, MSPs and the effects of tokenization.

As any research endeavour, limitations beset our work. First, we restricted our token valuation analysis to simple input factors. Since the blockchain is still a relatively young technology, only few financial instruments address this technology. Future research should build a more complex token valuation method to take more aspects of monetary theory into account as soon as sufficient empirical information is available. Such future research could incorporate other aspects of financial theory or shareholder incentives, e.g., by applying option pricing theory, or financial theory on shareholder incentives. Second, we assumed that the PPP holds to model the token value. However, PPP is only given for a good or service that is tradeable with foreign markets (Balassa 1964). As the blockchain technology holds the potential for new business models, it is likely that the good or service is only accessible on the blockchain-based platform and cannot be traded outside the platform (i.e., single-homing). This strengthens the necessity for more complex valuation methods in future research. Third, we did not investigate the implications of declining user activity on token value. Future research should develop an economic equilibrium model for token valuation, putting an increase in user numbers with a decrease of user activities in 
relation. Fourth, we only focused on the buyer-side behaviour in the two-sided market. Future research should also assess the seller-side and derive implications of an increase in token value on the behaviour of a seller. In addition, further considerations on risk attitudes of the buyers and sellers towards the token value upside potential would be desirable.

Our findings regarding the effects of a financial incentive on platform adoption can also stimulate further empirical studies. We suggest an inhibition of platform usage and provide a foundation for future research dedicated to proving an effect of a change in token value on platform adoption. As soon as sufficient long-term data development of token exchange rates and platform growth is available, research should shift its focus to this empirical effect. These studies may generate valuable insights for practice and help to identify sustainable token designs. Future research should also focus on developing token designs that accelerate platform growth without the contradictory effect of current token designs.

Funding Information Open Access funding provided by Projekt DEAL.

Open Access This article is licensed under a Creative Commons Attribution 4.0 International License, which permits use, sharing, adaptation, distribution and reproduction in any medium or format, as long as you give appropriate credit to the original author(s) and the source, provide a link to the Creative Commons licence, and indicate if changes were made. The images or other third party material in this article are included in the article's Creative Commons licence, unless indicated otherwise in a credit line to the material. If material is not included in the article's Creative Commons licence and your intended use is not permitted by statutory regulation or exceeds the permitted use, you will need to obtain permission directly from the copyright holder. To view a copy of this licence, visit http://creativecommons.org/licenses/by/4.0/.

\section{References}

Armstrong, M. (2006). Competition in two-sided markets. The Rand Journal of Economics, 37(3), 668-691. https://doi.org/10.1111/j. 1756-2171.2006.tb00037.x.

Aru, I. (2018). The Fundamental Principles of Utility Tokens in the Blockchain Ecosystem. Retrieved from www.ccn.com/ fundamental-principles-utility-tokens-blockchainhttps://www.ccn. com/fundamental-principles-utility-tokens-blockchain-ecosystem/

Asanov, P. (2018). Asset-Backed vs. Utility Tokens: Understanding the Pros and Cons. Retrieved from https://medium.com/equitytoken/ asset-backed-vs-utility-tokens-understanding-the-pros-and-consca4d1cf41432

Bachmann, N., Drasch, B. J., Miksch, M., \& Schweizer, A. (2019). Dividing the ICO jungle: Extracting and evaluating design archetypes. In Proceedings of the 14th international conference on Wirtschaftsinformatik. Germany: Siegen.

Bakos, Y., \& Halaburda, H. (2019). The role of cryptographic tokens and ICOs in fostering platform adoption. CESifo Working Paper(7752). Retrieved from http://hdl.handle.net/10419/201978

Balassa, B. (1964). The purchasing-power parity doctrine: A reappraisal. Journal of Political Economy, 72(6), 584-596. https://doi.org/10. 1086/258965.

Baldwin, C. Y., \& Clark, C. B. (2000). Design Rules, Vol. 1: The Power of Modularity. Cambridge, Massachusetts: MIT Press.
Baldwin, C. Y., \& Woodard, C. J. (2009). The architecture of platforms: A unified view. In A. Gawer (Ed.), Platforms, markets and innovation (pp. 19-44). Cheltenham, UK, Northhampton, MA, USA: Edward Elgar Publishing.

Beaman, K. (2018). Valuing a Payment Utility Token: IvyKoin Case Study. Retrieved from https://medium.com/datadriveninvestor/ valuing-a-payment-utility-token-ivykoin-case-study-1abb7376b 964

Beck, R., Czepluch, J. S., Lollike, N., \& Malone, N. (2016). Blockchain - the gateway to trust-free cryptographic transactions. Proceedings of the 24th European conference on information systems, Istanbul, Turkey.

Belleflamme, P., \& Peitz, M. (2019). Platform competition: Who benefits from multihoming? International Journal of Industrial Organization, 64, 1-26. https://doi.org/10.1016/j.jindorg.2018.03.014.

Boreiko, D., \& Sahdev, N. K. (2018). To ICO or not to ICO - Empirical analysis of initial coin offerings and token sales. SSRN Electronic Journal. https://doi.org/10.2139/ssrn.3209180.

Brand, K. (2017). Digital Business Models and Platform Economy. Retrieved from https://kubraconsult.blog/2017/11/04/digitalbusiness-models-and-platform-economy/

Brousseau, E., \& Penard, T. (2007). The economics of digital business models: A framework for analyzing the economics of platforms. Review of Network Economics, 6(2), 81-114. https://doi.org/10. 2202/1446-9022.1112.

Burniske, C. (2017). Cryptoasset Valuations. Retrieved from https:// medium.com/@cburniske/cryptoasset-valuations-ac83479ffca7

Buterin. (2014). A next-generation smart contract and decentralized application platform. Whitepaper. Retrieved from https://github.com/ ethereum/wiki/wiki/White-Paper\#decentralized-autonomousorganizations

Buterin, V. (2017). On Medium-of-Exchange Token Valuations. Retrieved from https://vitalik.ca/general/2017/10/17/moe.html

Caillaud, B., \& Julien, B. (2003). Chicken \& egg: Competition among intermediation service providers. The Rand Journal of Economics, 34(2), 309-328.

Catalini, C., \& Gans, J. (2016). Some simple economics of the Blockchain. SSRN Electronic Journal. https://doi.org/10.3386/w22952.

Chen, Y. (2018). Blockchain tokens and the potential democratization of entrepreneurship and innovation. Business Horizons, 61(4), 567575. https://doi.org/10.1016/j.bushor.2018.03.006.

Chod, J., Trichakis, N., \& Yang, S. A. (2019). Platform Tokenization: Financing, Governance, and Moral Hazard. SSRN Electronic Journal. https://doi.org/10.2139/ssrn.3459794.

Choi, J. P. (2010). Tying in two-sided markets with multi-homing. The Journal of Industrial Economics, 58(3), 607-626. https://doi.org/10. 1111/j.1467-6451.2010.00426.x.

Chuen, K., David, L., Guo, L., \& Wang, Y. (2017). Cryptocurrency: A new investment opportunity? SSRN Electronic Journal. https://doi. org/10.3905/jai.2018.20.3.016.

Cong, L., Li, Y., \& Wang, N. (2018). Tokenomics: Dynamic adoption and valuation. SSRN Electronic Journal. https://doi.org/10.2139/ssrn. 3153860 .

Conley, J. P. (2017). Blockchain and the economics of crypto-tokens and initial coin offerings (no. VUECON-17-00008). Nashville, TN, USA. Retrieved from Vanderbilt University Department of Economics working papers website: http://www.accessecon.com/ includes/CountdownloadPDF.aspx?PaperID=VUECON-SUB-1700008

Corbet, S., Larkin, C. J., Lucey, B. M., \& Yarovaya, L. (2018). Kodakcoin: A Blockchain revolution or exploiting a potential Cryptocurrency bubble? SSRN Electronic Journal. https://doi.org/ $10.2139 / \mathrm{ssrn} .3140551$.

Dale, O. (2018). What is tokenization? Democratizing Ownership \& Real-World Assets on the Blockchain. Retrieved from https:// blockonomi.com/tokenization-blockchain/ 
de Reuver, M., Sørensen, C., \& Basole, R. C. (2018). The digital platform: A research agenda. Journal of Information Technology, 33(2), 124-135. https://doi.org/10.1057/s41265-016-0033-3.

Dixon, C. (2017). Crypto Tokens: A Breakthrough in Open Network Design. Retrieved from https://medium.com/@cdixon/cryptotokens-a-breakthrough-in-open-network-design-e600975be2ef

Ehrsam, F. (2016a). Blockchain Tokens and the dawn of the Decentralized Business Model. Retrieved from https://blog. coinbase.com/app-coins-and-the-dawn-of-the-decentralizedbusiness-model-8b8c951e734f

Ehrsam, F. (2016b). How to raise money on a Blockchain with a token. Retrieved from How to Raise Money on a Blockchain with a Token.

Erickson, K. (2018). The Future Of Network Effects: Tokenization and the End of Extraction. Retrieved from https://medium.com/publicmarket/the-future-of-network-effects-tokenization-and-the-end-ofextraction-a0f895639ffb

Evans, D. S. (2003). The antitrust economics of multi-sided platform markets. Yale journal on regulation, 20(325), 325-381.

Evans, D. S. (2009). How catalysts ignite: The economics of platformbased start-ups. In A. Gawer (Ed.), Platforms, markets and innovation (pp. 99-128). Cheltenham, UK, Northhampton, MA, USA: Edward Elgar Publishing.

Evans, D. S. (2014). Economic aspects of Bitcoin and other decentralized public-ledger currency platforms. University of Chicago CoaseSandor Institute for Law \& Economics, Research Paper No. 685. doi:https://doi.org/10.2139/ssrn.2424516.

Evans, A. (2018). Value, Velocity and Monetary Theory - A New Approach to Cryptoasset Valuations. Retrieved from https:// medium.com/blockchannel/on-value-velocity-and-monetarytheory-a-new-approach-to-cryptoasset-valuations-32c9b22e3b6f

Evans, P. C., \& Gawer, A. (2016). The rise of the platform enterprise: A global survey. New York, NY, USA. Retrieved from The Center for Global Enterprise website: https://www.thecge.net/app/uploads/ 2016/01/PDF-WEB-Platform-Survey_01_12.pdf

Evans, D. S., Hagiu, A., \& Schmalensee, R. (2008). Invisible engines: How software platforms drive innovation and transform industries. Boston, MA, USA: The MIT Press

Foley, S., Karlsen, J. R., \& Putniņš, T. J. (2019). Sex, drugs, and Bitcoin: How much illegal activity is financed through Cryptocurrencies? The Review of Financial Studies, 32(5), 1798-1853. https://doi. org/10.1093/rfs/hhz015.

Frenkel, J. A. (1977). Flexible exchange rates and stabilization policy. In J. Herin, A. Lindbeck, \& J. Myhrman (Eds.), Flexible exchange rates and stabilization policy (pp. 68-92). London, UK: Palgrave Macmillan.

Fridgen, G., Regner, F., Schweizer, A., \& Urbach, N. (2018). Don't slip on the initial coin offering (ICO) - A taxonomy for a blockchainenabled form of Crowdfunding. In Proceedings of the 26the European conference on information systems. Portsmouth: UK.

Friedman, M. (2017). Quantity theory of money. In S. N. Durlauf \& L. E. Blume (Eds.), The new Palgrave dictionary of economics (pp. 5305-5327). London, UK: Palgrave Macmillan.

Gawer, A. (2014). Bridging differing perspectives on technological platforms: Toward an integrative framework. Research Policy, 43(7), 1239-1249. https://doi.org/10.1016/j.respol.2014.03.006.

Glaser, F. (2017). Pervasive decentralisation of digital infrastructures: A framework for Blockchain enabled system and use case analysis. Proceedings of the 50th Hawaii International Conference on System Sciences, Waikoloa, HI, USA. Doi:https://doi.org/10.24251/ HICSS.2017.186.

Haffke, L., \& Fromberger, M. (2018). ICO Market Report 2017. Performance analysis of initial coin offerings (presentation slides). SSRN Electronic Journal. https://doi.org/10.2139/ssrn.3309271.

Hagiu, A. (2007). Multi-Sided Platforms: From Microfoundations to Design and Expansion Strategies. SSRN Electronic Journal. doi: https://doi.org/10.2139/ssrn.955584.
Hagiu, N., \& Wright, J. (2015). Multi-sided platforms. International Journal of Industrial Organization, 43, 162-174. Retrieved from https://doi.org/10.1016/j.ijindorg.2015.03.003

Hargadon, A. (2008). How breakthroughs happen: The surprising truth about how companies innovate ([Repr.]). Boston, MA, USA: Harvard Business School Press.

Harrigan, M., Shi, L., \& Illum, J. (2018). Airdrops and privacy: A case study in cross-Blockchain analysis. In IEEE International Conference on Data Mining Workshops (pp. 63-70). Retrieved from https://arxiv.org/pdf/1809.05360.pdf

Henderson, R. M., \& Clark, K. B. (1990). Architectural innovation: the reconfiguration of existing product technologies and the failure of established firms. Administrative Science Quaterly, 35, 9-30.

Henfridsson, O., Mathiassen, L., \& Svahn, F. (2014). Managing technological change in the digital age: The role of architectural frames. Journal of Information Technology, 29(1), 27-43. https://doi.org/10. 1057/jit.2013.30.

ICOscoring. (2018). Types of tokens. The four mistakes beginner cryptoinvestors make. Retrieved from https://medium.com/swlh/types-oftokens-the-four-mistakes-beginner-crypto-investors-makea76b53be5406

Kaal, W. A. (2018). Crypto economics - the top 100 token models compared. SSRN Electronic Journal. https://doi.org/10.2139/ssrn. 3249860 .

Karnjanaprakorn, M. (2017). Token Network Effects - New business model for a decentralized web. Retrieved from https://medium. freecodecamp.org/token-network-effects-a-new-business-modelfor-a-decentralized-web-6cde8b4e862

Katalyse.io. (2018). Security tokens vs. Utility Tokens - How different are they. Retrieved from https://cryptodigestnews.com/securitytokens-vs-utility-tokens-how-different-are-they-8a439c73e616.

Katz, M. L., \& Shapiro, C. (1985). Network externalities, competition, and compatibility. The American Economic Review, 75(3), 424-440.

Kenney, M., \& Zysman, J. (2016). The rise of the platform economy. Issues in Science and Technology, 32(3), 61-69.

Kilroe, J. (2017). Velocity of Tokens. Retrieved from https://medium.com/ newtown-partners/velocity-of-tokens-26b313303b77

King, S., \& Nadal, S. (2012). PPCoin: Peer-to-Peer Crypto-Currency with Proof-of-Stake. Retrieved from https://peercoin.net/assets/ paper/peercoin-paper.pdf

Kristoufek, L. (2013). BitCoin meets Google trends and Wikipedia: Quantifying the relationship between phenomena of the internet era. Scientific Reports, 3(3415). https://doi.org/10.1038/srep03415.

Kuo Chuen, D. L. (2017). Fintech Tsunami: Blockchain as the Driver of the Fourth Industrial Revolution. SSRN Electronic Journal. https:// doi.org/10.2139/ssrn.2998093.

Lee, J., Li, T., \& Shin, D. (2018). The wisdom of crowds and information cascades in FinTech: Evidence from Initial Coin Offerings. SSRN Electronic Journal. https://doi.org/10.2139/ssrn.3195877.

Li, J., \& Mann, W. (2018). Initial coin offering and platform building. SSRN Electronic Journal. https://doi.org/10.2139/ssm.3088726.

Liebowitz, S. J., \& Margolis, S. E. (1994). Network externality: An uncommon tragedy. Journal of Economic Perspectives, 8(2), 133-150. https://doi.org/10.1257/jep.8.2.133.

Lindman, J., Tuunainen, V. K., \& Rossi, M. (2017). Opportunities and risks of Blockchain technologies: A research agenda. Proceedings of the 50th Hawaii International Conference on System Sciences, Waikoloa, HI, USA. Doi:https://doi.org/10.24251/HICSS.2017.185.

Michael, J. W., Cohn, A., \& Butcher, J. R. (2018). BlockChain technology. Retrieved from www.steptoe.com/images/content/1/7/ v2/171967/LIT-FebMar18-Feature-Blockchain.pd

Momtaz, P. P. (2019). The pricing and performance of Cryptocurrency. The European Journal of Finance, 35, 1-14. https://doi.org/10. 1080/1351847X.2019.1647259.

Nakamoto, S. (2008). Bitcoin: A peer-to-peer electronic cash system. Retrieved from https://bitcoin.org/bitcoin.pdf 
Newcomb, S. (1913). Principles of political economy. New York, NY, USA: Harper \& Brothers.

Oliveira, L., Zavolokina, L., Bauer, I., \& Schwabe, G. (2018). To token or not to token: Tools for understanding Blockchain tokens. In Proceedings of the 39th international conference on information systems. San Francisco: CA, USA.

Oren, S. S., \& Smith, S. A. (1981). Critical mass and tariff structure in electronic communications markets. The Bell Journal of Economics, 12(2), 467-487. https://doi.org/10.2307/3003567.

Parker, G., \& van Alstyne, M. W. (2009). Six challenges in platform licensing and open innovation. Communications \& strategies(74), 17-36.

Parker, G., \& van Alstyne, M. (2018). Innovation, openness, and platform control. Management Science, 64(7), 3015-3032. https://doi.org/10. 1287/mnsc.2017.2757.

Pietrewicz, L. (2017). Emerging trends in entrepreneurial finance: The rise of ICOs. Retrieved from www.researchgate.net/profile/Leslaw Pietrewicz/publication/322197042_Emerging_trends_in_ entrepreneurial finance The rise of ICOs/links/ 5a4ab049aca272d294646e6b/Emerging-trends-in-entrepreneurialfinance-The-rise-of-ICOs.pd

Reid, F., \& Harrigan, M. (2013). An analysis of anonymity in the Bitcoin system. In Y. Altshuler, Y. Elovici, A. Cremers, N. Aharony, \& A. Pentland (Eds.), Security and privacy in social networks. New York, NY, USA: Springer.

Rochet, J.-C., \& Tirole, J. (2003). Platform competition in two-sided markets. Journal of the European Economic Association, 1(4), 990-1029. https://doi.org/10.1162/154247603322493212.

Schweizer, A., Schlatt, V., Urbach, N., \& Fridgen, G. (2017). Unchaining social businesses - Blockchain as the basic Technology of a Crowdlending Platform. In Proceedings of the 38th international conference on information systems. Seoul: South Korea.

Shapiro, C., \& Varian, H. R. (1999). Information rules: A strategic guide to the network economy. Boston, MA, USA: Harvard Business School Press.

Shoshitaishvili, Y., Invernizzi, L., Doupe, A., \& Vigna, G. (2014). Do you feel lucky? In Y. Cho, S. Y. Shin, S. Kim, C.-C. Hung, \& J. Hong (Eds.), Proceedings of the 29th annual ACM symposium on applied computing - SAC '14 (pp. 1649-1656). New York, New York, USA: ACM Press.

Smith, A., \& Weismann, M. F. (2014). Are you ready for digital currency? The Journal of Corporate Accounting and Finance, 26(1), 17-21. https://doi.org/10.1002/jcaf.21999.

Swan, M. (2015). Blockchain: Blueprint for a new economy (First ed.). Sebastopol, CA, USA: O'Reilly Media, Inc..

Szabo, N. (1997). Formalizing and securing relationships on public networks. First Monday, 2(9). https://doi.org/10.5210/fm.v2i9.548.

Tsoulfidis, L. (2008). International encyclopedia of the social sciences. In W. A. Darity (Ed.), International Enciclopedia of the social sciences (pp. 659-661). Detroit, MI, USA: Thomson Gale.
Urgo, J., Lestan, M., \& Khoriaty, A. (2017). district0x network: A cooperative of decentralized marketplaces and communities. Powered by Ethereum, Aragon, and IPFS. Retrieved from https://district0x.io/ docs/district0x-whitepaper.pdf

van Hout, M. C., \& Bingham, T. (2014). Responsible vendors, intelligent consumers: Silk road, the online revolution in drug trading. The International Journal on Drug Policy, 25(2), 183-189. https://doi. org/10.1016/j.drugpo.2013.10.009.

van Niekerk, M., \& van der Veer, R. (2018). databroker dao - Global market for local data. Retrieved from https://databrokerdao.com/wpcontent/uploads/2018/09/whitepaper_databrokerdao.pdf

Walter, M. (2017). How Blockchain will transform the platform economy-Part 1. Retrieved from https://medium.com/platforminnovation-kit/how-blockchain-will-transform-the-platformeconomy-part-1-e5994de8663d

Weitzman, M. L. (1998). Recombinant growth. The Quarterly Journal of Economics, 113(2), 331-360. https://doi.org/10.1162/ 003355398555595.

Wesley, D. (2018). Utility Tokens vs Security Tokens: What the Future of ICOs Will Look Like. Retrieved from https://hackernoon.com/utilitytokens-vs-security-tokens-what-the-future-of-icos-will-look-likefaa9017e8aa8

Wilmoth, J. (2018). The Difference Between Utility Tokens and Equity Tokens. Retrieved from https://strategiccoin.com/difference-utilitytokens-equity-tokens/

Woodford, M. (2011). Interest and prices: Foundations of a theory of monetary policy. New Jersey: Princeton University Press. Retrieved from https://ebookcentral.proquest.com/lib/gbv/detail. action?docID $=617424$

Xu, X., Weber, I., Staples, M., Zhu, L., Bosch, J., Bass, L., . . Rimba, P. (2017). A taxonomy of Blockchain-based Systems for Architecture Design. In IEEE International Conference on Software Architecture (pp. 243-252).

Zawistowski, J., Janiuk, P., \& Regulski, A. (2016). The golem project Crowdfunding. Whitepaper. Retrieved from https://golem.network/ crowdfunding/Golemwhitepaper.pdf

Zheng, Z., Xie, S., Dai, H., Chen, X., \& Wang, H. (2017). An overview of Blockchain technology: Architecture, consensus, and future trends. In 6th IEEE International Congress on Big Data (pp. 557-564).

Zhu, F., \& Iansiti, M. (2012). Entry into platform-based markets. Strategic Management Journal, 33(1), 88-106. https://doi.org/10. 1002/smj.941.

Publisher's note Springer Nature remains neutral with regard to jurisdictional claims in published maps and institutional affiliations. 